\section{Case Reports in Oncology}

\title{
Rare Paravertebral and Skull Base Metastases in Prostate Cancer
}

\author{
Gbeminiyi Samuel $^{b} \quad$ Amir Isbell $^{a} \quad$ Onyekachi Ogbonna ${ }^{c}$ Hasan Iftikhar $^{b}$ \\ Susmita Sakrutic ${ }^{c}$ Adebayo Atanda $^{b}$ Raj P. Manchandani ${ }^{c}$ \\ ${ }^{a}$ Howard University College of Medicine, Washington, DC, USA; ${ }^{b}$ Department of \\ Internal Medicine, Howard University Hospital, Washington, DC, USA; ${ }^{C}$ Division of \\ Hematology and Oncology, Department of Internal Medicine, Howard University \\ Hospital, Washington, DC, USA
}

\section{Keywords}

Prostate cancer - Paravertebral metastasis · Skull base metastasis - Mixed sclerotic and lytic lesions

\begin{abstract}
Prostate cancer is the most commonly diagnosed visceral cancer in the United States. A majority of cases exhibit an insidious course and nonaggressive tumor behavior. Prostate cancer can manifest as lesions which remain localized, regionally invading or metastasize to lymph nodes, bones, and lungs. Here, we report a unique case of metastatic prostate cancer to the right upper mediastinum, presenting as a paravertebral mass within 2 years of initial tissue diagnosis. Paravertebral spread has not been described for prostate cancer, and herein, we discuss the clinical presentation, diagnostic workup, and possible therapeutic options available in light of the literature.

(C) 2016 The Author(s)

Published by S. Karger AG, Basel
\end{abstract}

\section{Introduction}

Prostate cancer is the most commonly diagnosed visceral cancer [1], and the second leading cancer and cause of cancer deaths in men in the United States [2]. Autopsy series have detected prostate cancer in about 1 out of 3 of men aged 55 years and in about 3 out of 
5 men by age 80 years [3]. Disease survival is prognosticated by many factors, and in particular by tumor extent at the time of diagnosis. Five-year relative survival in men with localized disease is $100 \%$, compared to $<30 \%$ among those with metastases. The most common sites of metastasis are the bone, lung, and liver [4]. Paravertebral metastasis from prostate cancer is rare, with only a limited number of cases reported. We discuss an interesting case of prostate cancer with paravertebral metastasis and present an approach to management.

\section{Case Presentation}

The patient was a 55-year-old African-American man with a history of prostate cancer (diagnosed via core needle biopsy 2 years prior). At that time, the patient had 6 specimens obtained from different prostatic tissue locations submitted for histopathology. Four out of the 6 specimens showed benign prostate tissue in the left apex, right apex, left mid, and left base. The remaining 2 specimens showed prostatic adenocarcinoma in the right base and right mid zones, respectively: a Gleason score of $9(5+4)$ was noted in 4 out of 4 submitted core segments from the right base, and a Gleason score of $8(4+4)$ in 3 out of 3 core segments from the right mid zone. No therapy (chemotherapy, hormonal, or radiation) was implemented prior to presentation to our facility. He reported previously treated Lyme disease and chronic kidney disease stage 3, and complained of right neck pain that had started after a mechanical fall. He denied any trauma to the head, loss of consciousness, syncopal symptoms, any weakness in his lower extremities, unsteady gait or numbness, or tingling in his extremities. The patient also reported recent onset of coughing spells associated with drinking of fluids.

MRI of the thoracic spine disclosed significant compression of the T4 vertebral body with mild associated cord compression, and diffuse patchy enhancement throughout the thoracic spine. Initial CT scans revealed no acute bony trauma, but showed multiple destructive lytic lesions and partial sclerosis in the lower calvarium (Fig. 1), both clavicles, and the lower cervical and thoracic vertebra (Fig. 2). A paravertebral mass in the right upper mediastinum was also visualized (Fig. 3). Subsequent MRI images of the brain with and without contrast displayed a right-sided, $1.3 \times 4.3 \times 2.5 \mathrm{~cm}$, intermediate-signal mass at the base of the skull in both the T1- and T2-weighted sequences. Chest X-rays revealed a small right lung apical opacity with probable destruction of adjacent first and second ribs. Further workup showed small sclerotic densities in the left sacrum and right iliac bone, assessed as metastasis. Multiple myeloma workup, including serum and urine protein electrophoresis, immunofixation, beta- 2 microglobulin, and serum free light chain quantification, was negative. The serum PSA level was elevated at $994.47 \mathrm{ng} / \mathrm{ml}$.

To further assess the extent of disease, a bone scan was obtained after administering $26 \mathrm{mCi}$ of technetium-99m methylene diphosphonate intravenously. This revealed multiple foci of abnormally increased tracer accumulation involving multiple ribs bilaterally as well as the cervical, thoracic, and lumbar spine (Fig. 4). Small foci of increased tracer accumulation were also noted in the humeral shafts, left proximal femur, and left mid femoral shaft. MRI of the cervical and thoracic spine with and without contrast revealed diffuse, patchy, bony metastases and a paraspinal mass on the right, extending from $\mathrm{T} 1$ to $\mathrm{T} 4$ and measuring approximately $7 \mathrm{~cm}$ in craniocaudal dimension, with significant compression of the T4 vertebral body with mild associated cord compression (Fig. 5). MRI of the brain also revealed a contrast-enhancing mass at the base of the skull on both the T1 and FLAIR sequences (Fig. 6). 
The patient's symptoms (choking spells and neck pain) were attributed to cord compression as evident on MRI imaging, and he was started on dexamethasone for symptom relief. He was also started on 6 cycles of chemotherapy with Taxotere in addition to intramuscular leuprolide for treatment of his prostate cancer, given the extensive burden of his disease. Radiation oncology was consulted for consideration of radiotherapy to the lesion at the base of his skull and the paravertebral mass. They formulated a treatment plan of $2.5 \mathrm{~Gy}$ per fraction doses of external beam radiation to the skull base in 14 fractions for a total dose of $35 \mathrm{~Gy}$, and $2.5 \mathrm{~Gy}$ per fraction doses of radiation to the paravertebral mass in 14 fractions for a total dose of $35 \mathrm{~Gy}$. The patient's coughing paroxysms associated with swallowing resolved after just 2 sessions of radiotherapy.

The patient has had 4 cycles of chemotherapy thus far and is responding well to treatment. Restaging/surveillance imaging will be obtained once he has completed 6 cycles of chemotherapy.

\section{Discussion}

Prostate cancer is the second leading cause of cancer in men in the United States [2], and an important cause of cancer-related deaths in this demographic group. In particular, African-American men were found to have an incidence of 203.5 cases per 100,000 between 2009 and 2013 [5]. This is higher than the incidence in Caucasians (121.9), Hispanics (106.9), and Asians/Pacific Islanders (68.9) [5]. Blacks were also found to be more than twice as likely to die of prostate cancer as men belonging to other races [5].

This disparity in outcome is likely multivariate, and while the role of demographics and race remains to be better understood, investigating tumor cytogenetics offers a promising method to predict disease aggressiveness and outcomes. Genome-wide association studies and meta-analyses have shed light onto susceptibility loci for prostate cancer, including single nucleotide polymorphisms that may portend disease aggressiveness [6-10]. The advent of genome-wide association studies has identified 78 susceptibility loci (single nucleotide polymorphisms) associated with risk of prostate cancer [10]. While further studies are required to make them viable tools for risk stratification in clinically useful ways, these gene loci offer specific regions of interest for new research to focus on.

A puzzling piece in this clinical case was the actual route of metastasis to the mediastinum. In this regard, prior autopsy studies spanning over 19,000 cases in men, carried out between 1967 and 1995, identified 1,589 cases of prostate cancer and considered likely routes of metastasis [4]. Roughly one-third of prostate cancer cases were found to have hematogenous spread. Of these, $90 \%$ metastasized to the bone, $46 \%$ to the lung, $25 \%$ to the liver, $21 \%$ to the pleura, and $13 \%$ to the adrenals. This study reiterated the classical hematogenous spread via the vena cava, but also identified upward metastatic spread along the spinal veins after metastasization to the lumbar spine, which was hypothesized to occur early in the metastatic process [4]. The venous vertebral plexus was described fully in 1940 [11] and extends from the sacrum to the cranium. Our patient not only had a rare paravertebral mass, but also a skull base mass; this aberrant metastasis may be explained by the role of the vertebral vein system, as described by Batson in 1940 [11].

A few cases of CNS spread have been described. Our patient had signs of compression demanding immediate intervention barring cerebrospinal fluid analysis, and hence the important clinical question about whether or not there was leptomeningeal involvement was left unanswered. There have only been a few cases of leptomeningeal metastasis from pros- 
tate cancer reported in the literature $[12,13]$. The common signs for leptomeningeal involvement described were lower extremity weakness, neck pain, back pain, and/or sensory loss in a segmental or dermatomal distribution. Of the aforementioned signs, our patient only presented with neck pain. To the best of our knowledge, our case appears to be the first cited case of paravertebral metastasis from prostate cancer. The only mimic in the literature was a case of paravertebral schwannoma simulating prostate cancer metastasis [14].

The medical management for our patient was based on the current standard of care for metastatic prostate cancer. Given his extensive burden of visceral disease from metastases, we offered him systemic chemotherapy with Taxotere for 6 cycles in addition to androgen deprivation therapy (ADT) with leuprolide, based on a study which showed improved overall survival with using both chemotherapy and ADT compared with using ADT alone in patients with progressive androgen-stimulated prostate cancer and distant metastases [15]. Reviewing the literature for novel approaches to treating paravertebral spread did not yield any significantly different options. It did, however, lead to interesting perspectives about the spread of metastatic prostate cancer, specifically to neural structures by way of the spinal veins and plexus.

\section{Conclusion}

Paravertebral metastasis is uncommon in prostate cancer, and neurological symptoms such as coughing paroxysms associated with swallowing, as was noted in our patient, may clue one in to the diagnosis. Patients with extensive burden of visceral disease from prostate metastases may benefit from systemic chemotherapy in addition to androgen suppression with leuprolide. Strong consideration should be given to radiation therapy to promote local control where there is concern for cord compression or intractable bony pain.

\section{Acknowledgment}

The authors would like to acknowledge the help of Hasan Nabhani, MD, Department of Radiology, Howard University Hospital, and Fasil Tiruneh, MD, Department of Internal Medicine, Howard University Hospital.

\section{Statement of Ethics}

The authors have no ethical conflicts to disclose.

\section{Disclosure Statement}

The authors have no conflict of interest to declare. 


\section{References}

1 Siegel RL, Miller KD, Jemal A: Cancer statistics, 2016. CA Cancer J Clin 2016;66:7-30.

2 Torre LA, Bray F, Siegel RL, Ferlay J, Lortet-Tieulent J, Jemal A: Global cancer statistics, 2012. CA Cancer J Clin 2015;65:87-108.

-3 Bell KJL, Del Mar C, Wright G, Dickinson J, Glasziou P: Prevalence of incidental prostate cancer: a systematic review of autopsy studies. Int J Cancer 2015;137:1749-1757.

-4 Bubendorf L, Schöpfer A, Wagner U, Sauter G, Moch H, Willi N, Gasser TC, Mihatsch MJ: Metastatic patterns of prostate cancer: an autopsy study of 1,589 patients. Hum Pathol 2000;31:578-583.

$5 \quad$ SEER Cancer Statistics Review 1975-2013: Incidence and mortality by race/ethnicity. http://seer.cancer.gov/csr/1975_2013/results_merged/topic_race_ethnicity.pdf\#search=incidence+of +prostate+cancer+in+blacks (accessed September 29, 2016).

6 Bensen JT, Xu Z, Smith GJ, Mohler JL, Fontham ETH, Taylor JA: Genetic polymorphism and prostate cancer aggressiveness: a case-only study of 1,536 GWAS and candidate SNPs in African-Americans and European-Americans. Prostate 2013;73:11-22.

-7 Ishak MB, Giri VN: A systematic review of replication studies of prostate cancer susceptibility genetic variants in high-risk men originally identified from genome-wide association studies. Cancer Epidemiol Biomarkers Prev 2011;20:1599-1610.

8 Liu H, Wang B, Han C: Meta-analysis of genome-wide and replication association studies on prostate cancer. Prostate 2011;71:209-224.

-9 Chen R, Ren S, Sun Y: Genome-wide association studies on prostate cancer: the end or the beginning? Protein Cell 2013;4:677-686.

10 Mikropoulos C, Goh C, Leongamornlert D, Kote-Jarai Z, Eeles R: Translating genetic risk factors for prostate cancer to the clinic: 2013 and beyond. Future Oncol 2014;10:1679-1694.

11 Batson OV: The function of the vertebral veins and their role in the spread of metastases. Ann Surg 1940;112:138-149.

12 Cante D, Franco P, Sciacero P, Girelli G, Casanova Borca V, Pasquino M, Migliaccio F, Tofani S, Grassi L, Marra A, Ozzello F, La Porta MR, Ricardi U: Leptomeningeal metastasis from prostate cancer. Tumori 2013;99:6e-10e.

13 Orphanos G, Ardavanis A: Leptomeningeal metastases from prostate cancer: an emerging clinical conundrum. Clin Exp Metastasis 2010;27:19-23.

14 Rischpler C, Maurer T, Schwaiger M, Eiber M: Intense PSMA-expression using (68)Ga-PSMA PET/CT in a paravertebral schwannoma mimicking prostate cancer metastasis. Eur J Nucl Med Mol Imaging 2016;43:193-194.

15 Sweeney CJ, Chen YH, Carducci M, Liu G, Jarrard DF, Eisenberger M, Wong YN, Hahn N, Kohli M, Cooney MM, Dreicer R, Vogelzang NJ, Picus J, Shevrin D, Hussain M, Garcia JA, DiPaola RS: Chemohormonal therapy in metastatic hormone-sensitive prostate cancer. N Engl J Med 2015;373:737-746.

\section{G. Samuel and A. Isbell contributed equally.}




\section{Case Reports in Oncology}

\begin{tabular}{l|l}
\hline Case Rep Oncol 2016;9:738-746 \\
\hline DOI: $10.1159 / 000452327$ & $\begin{array}{l}\text { C } 2016 \text { The Author(s). Published by S. Karger AG, Basel } \\
\text { www.karger.com/cro }\end{array}$ \\
\hline
\end{tabular}

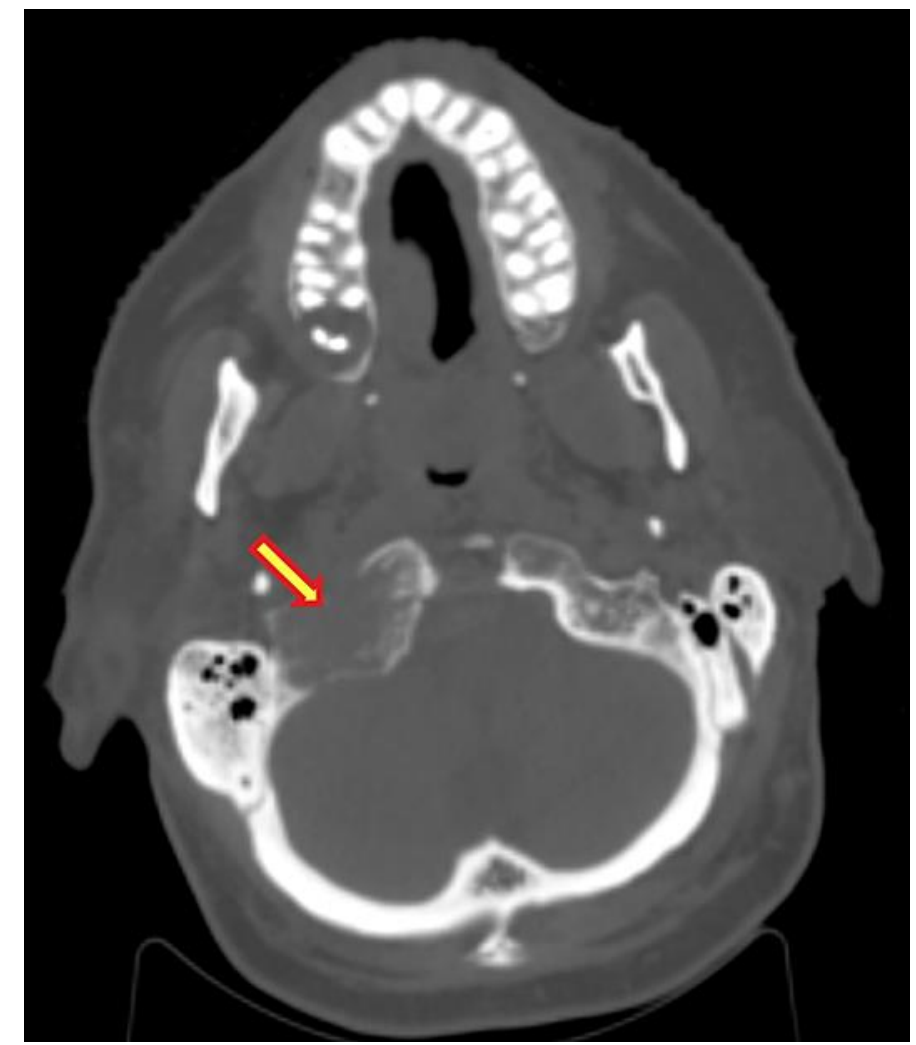

Fig. 1. CT of the head showing lytic and sclerotic lesions involving the base of the skull.
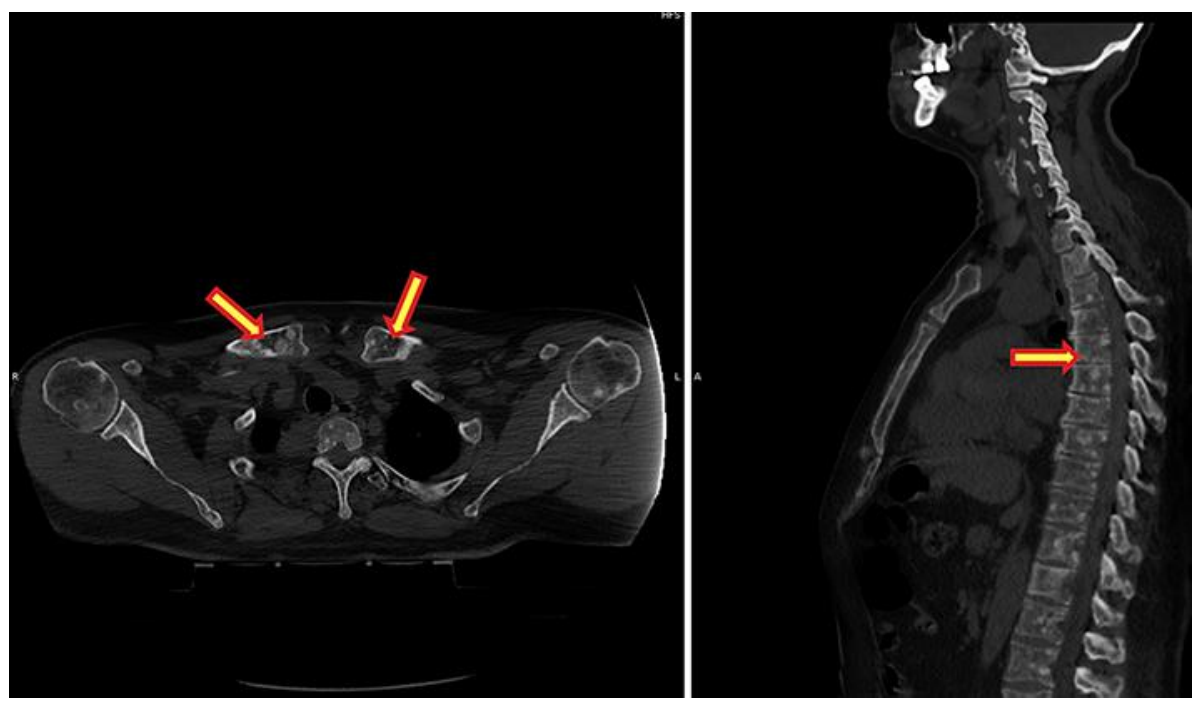

Fig. 2. CT scan showing lytic lesions and partial sclerosis in both clavicles as well as the lower cervical and thoracic vertebra. 


\section{Case Reports in Oncology}

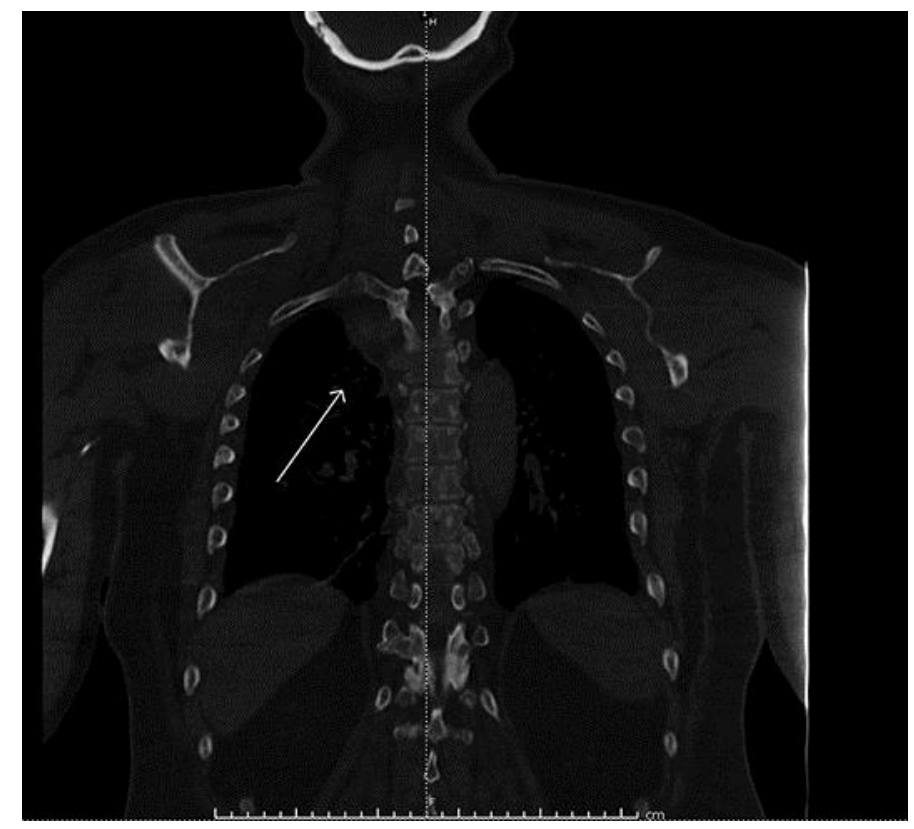

Fig. 3. MRI of the thoracic spine with and without contrast showing a paravertebral mass on the right, extending from $\mathrm{T} 1$ to $\mathrm{T} 4$, measuring approximately $7 \mathrm{~cm}$ in craniocaudal dimension. 


\section{Case Reports in Oncology

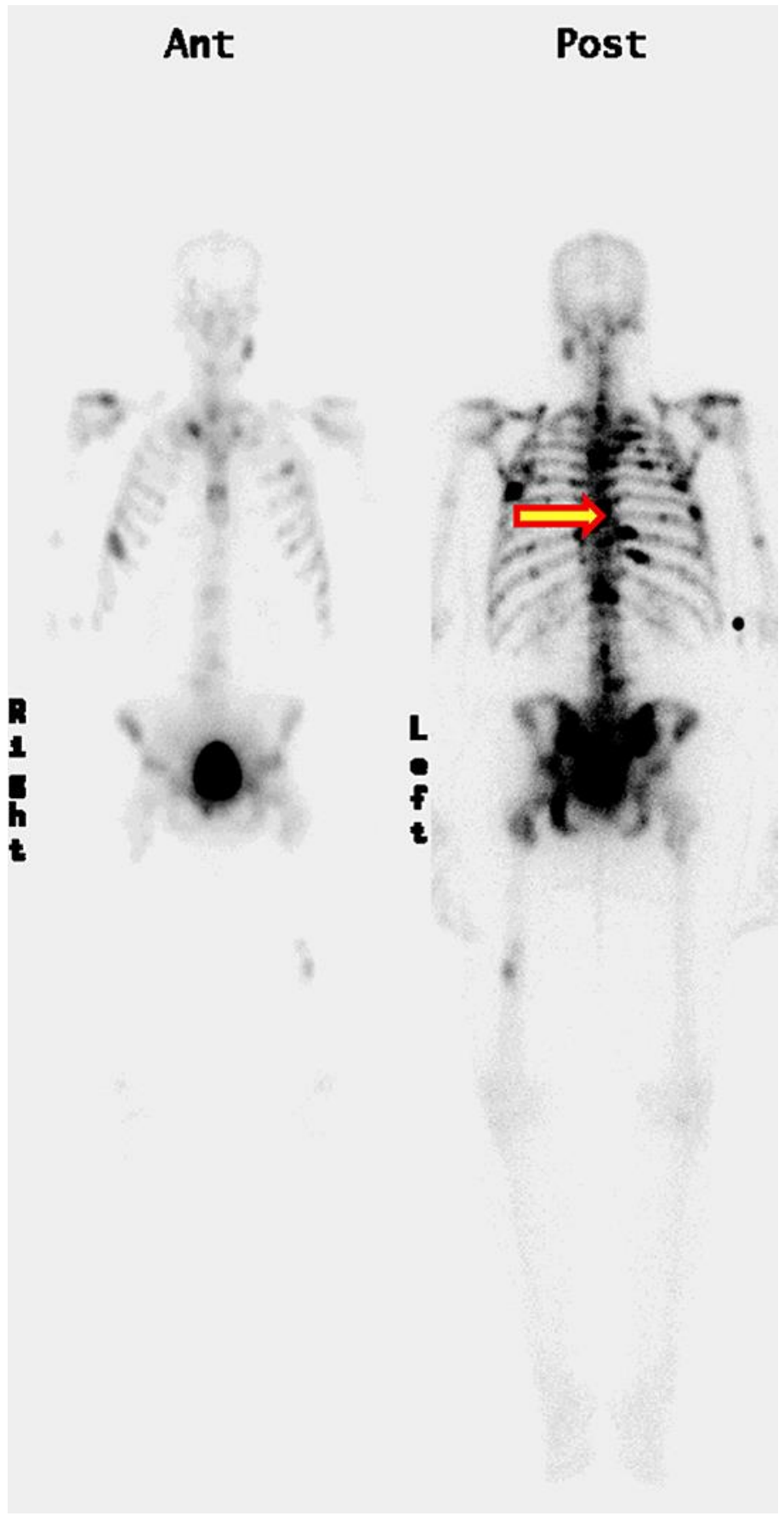

Fig. 4. Bone scan showing multiple bone metastases. 


\section{Case Reports in Oncology}
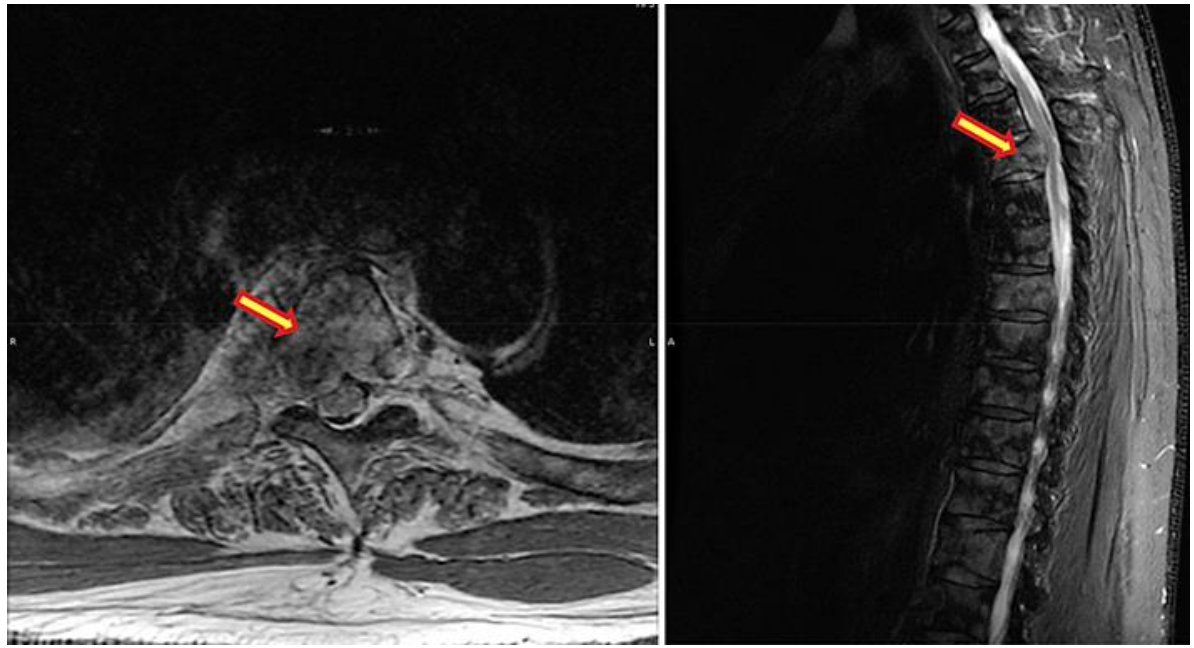

Fig. 5. MRI of the cervical and thoracic spine showing a paraspinal mass on the right, extending from T1 to T4.
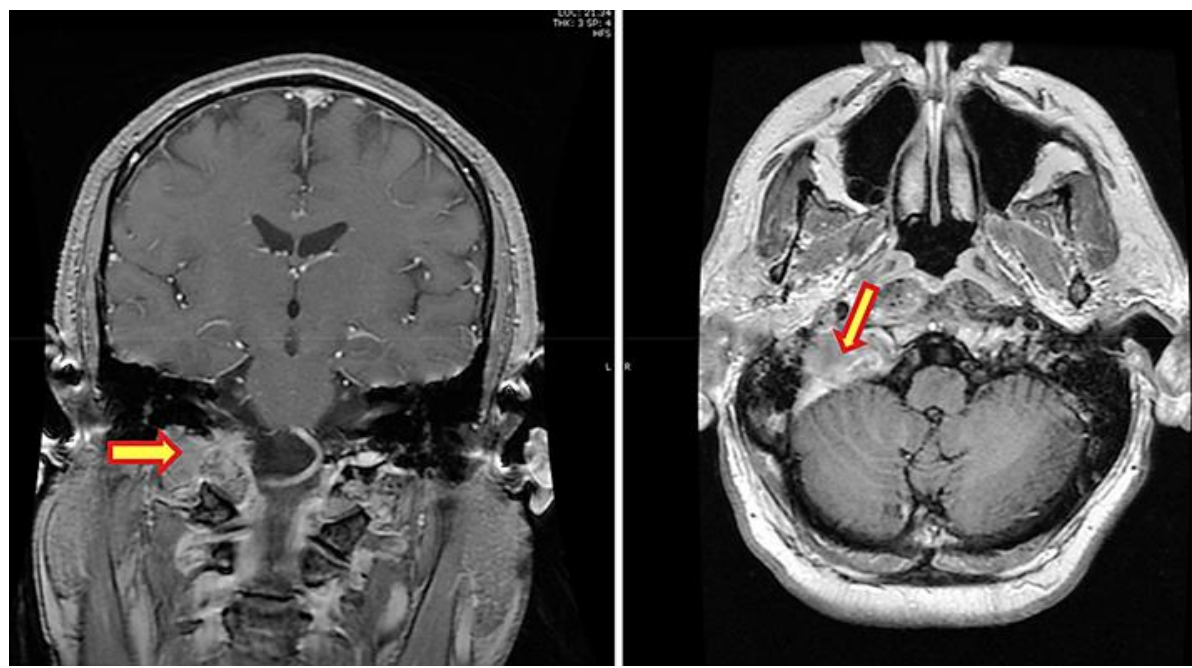

Fig. 6. MRI of the brain with contrast showing an enhancing mass at the base of the skull on the right on both the T1 and FLAIR sequences. 